\title{
Design of Plate Rolling Machine Based on Trio Motion Controller
}

\author{
Chun-You Zhang ${ }^{1, a}$, Da-Peng Liu ${ }^{2, b}$ \\ ${ }^{1}$ Inner Mongolia University for the Nationalities, College of Mechanical Engineeringe, TongLiao, \\ 028000, China; \\ ${ }^{2}$ NO.724 Research Institute under CSIC, Nanjing Jiangsu 210003, China; \\ awuxiaoqiang6006@163.com, bliudapeng@163.com
}

Keywords: Plate rolling machine, Trio motion controller, Openness, Control system.

\begin{abstract}
The development of industrial automation puts forward new requirements of plate rolling machine, traditional plate rolling machine has been unable to meet the market demand. In view of this situation, this paper a kind of control system of plate rolling machine is designed based on trio motion controller, the hardware and software of the system are introduced respectively. The system uses the modular design, it improves the openness and the expansibility of the system. Double CPU structure can guarantee the operating speed and control accuracy of the system, and it has some reference value.
\end{abstract}

\section{Introduction}

Plate rolling machine is a molding device according to the principle of the three points making the circle, the rotation of work roll and the position changing make the sheet metal continuous elastic plastic rolling to obtain a predetermined shape and precision [1]. With the continuous development of aviation, aerospace, shipbuilding, automobile, chemical and other industries, the demand of plate rolling machine is increasing, rolling precision requirement is also higher and higher [2]. The continuous development of China's manufacturing industry puts forward new requirements on the reliability, safety and automation of the plate rolling machine [3]. The original plate rolling machine uses high voltage control and manual operation mode of production, the strength of this production is very big, production efficiency is low [4]. With the development of CNC technology, the use of existing CNC technology is put into plate rolling machine. Machine working environment is relatively poor, there are shock, vibration and other unfavorable factors [5]. In view of the above situation, a kind of plate rolling machine is designed based on trio motion controller in this paper, the system adopts double CPU structure of "PC + motion controller", modular design is adopted for the system and improves the system stability and control precision.

\section{Hardware of the plate rolling machine}

Overall design: As shown in Figure 1, rolling machine is divided into mechanical part and control part, the motion control of mechanical part is through the control system. The motion controller is the core of the whole control system, after years of development, open CNC system has become the mainstream, it can meet the different requirements, with open and expansibility, the user can continue to improve the system on the basis, in order to further improve the openness of the system, open motion controller must be chosen, through contrast, in this paper, the trio motion controller is adopted, TRIO digital motion controller is a kind of digital motion controller developed based on micro processing technology, the precision is high. TRIO digital motion controller has good expansibility and it provides a variety of functional modules, it can configure according to the application requirements and it improves openness and portability of the system. 


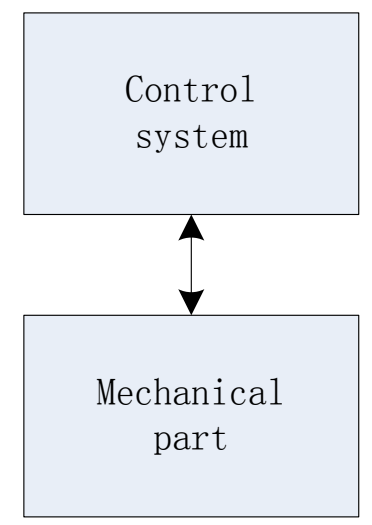

Figure1. Overall design

Mechanical part design: Plate rolling machine working principle follows three point making circle principle, in accordance with the principle of the plate rolling machine, plate rolling machine mechanical structure is shown in figure 2. Plate rolling machine adopts the most common form of three rollers, plate rolling machine mainly consists of a upper roller and two lower roller, they are driven by motors. Upper roller is driving roller, and the horizontal and vertical motion are driven respectively by hydraulic cylinder, in horizontal and vertical direction are respectively provided with a grating ruler, they are used to measure the displacement information and feedback the information to the system, and the lower roller is equipped with a rotary encoder, it is used for detection and the rotational position.

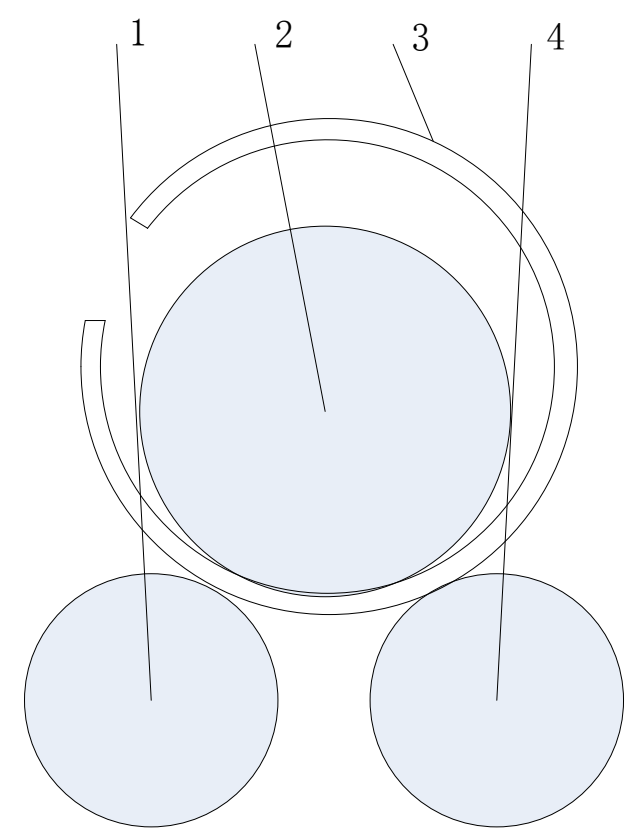

1. Left lower roller 2. Upper roller 3.Plate 4.Right lower roller Figure2. Mechanical part

Control system design: Plate rolling machine control system must also be according to the three-point principle to carry on the design, precise and flexible control of the plate rolling machine is required. Rolling machine control system is shown in figure 3. All actions are completed in the machine under the control of the system. Trio motion controller is the core of the system, it used for the management and control of other devices and receiving grating ruler and a rotary encoder feedback signal to the system, the system is through the signal detection and control of the motor to work. 


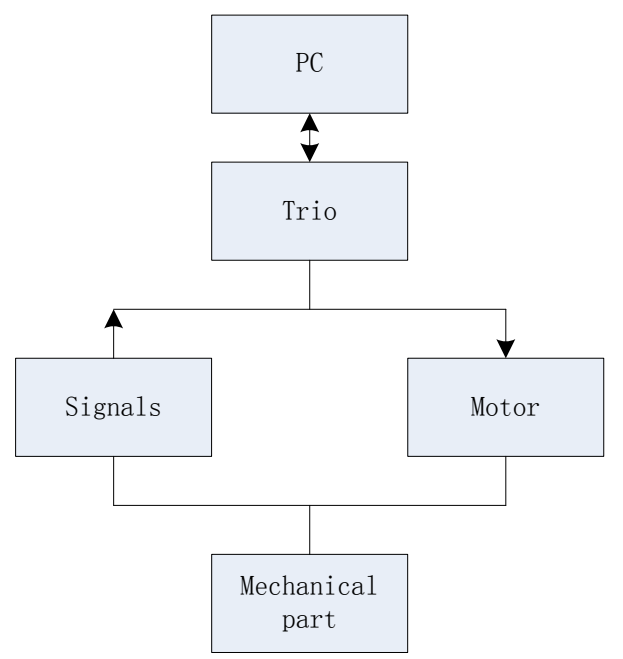

Figure 3. The control system design

\section{Software of the plate rolling machine}

Software structure design: The design of plate rolling machine control system takes an open design concept, as shown in Figure 3, according to the system hardware design, the software also takes modular design. The hardware is divided into upper and lower computer, and the software is designed in the upper and lower computer. The software of the upper computer is mainly used to complete the non-real time task, and the lower computer software is used to perform real-time tasks. Dual CPU structure can ensure the running speed of the system, upper computer program is developed on the PC based on Windows system, the programs of the lower computer is through the PC to write, the upper computer and the lower computer can communicate directly, in order to facilitate debugging and transmission.

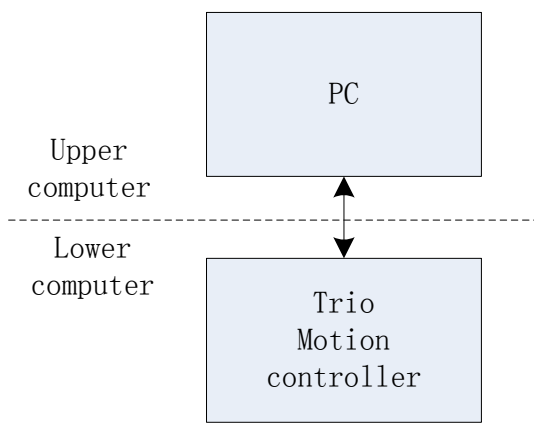

Figure4. Software structure

The upper computer program: Trio motion controller provides the interface of upper computer programming for the user, in order to further improve the friendliness of system interface, the upper computer program is on the Windows platform using Visual Basic language to design, as shown in Figure 4, the main functions of the upper computer software are: parameter setting, parameter display, programming and fault diagnosis. Parameter setting module is used to set the parameters of the controller and the motor, and ensure the smooth operation of the system; parameter display module is used for displaying the running state of the system. The program is used to write program for a motion controller; fault diagnosis can show the fault, and the fault can records, it is conducive to the follow-up treatment. 


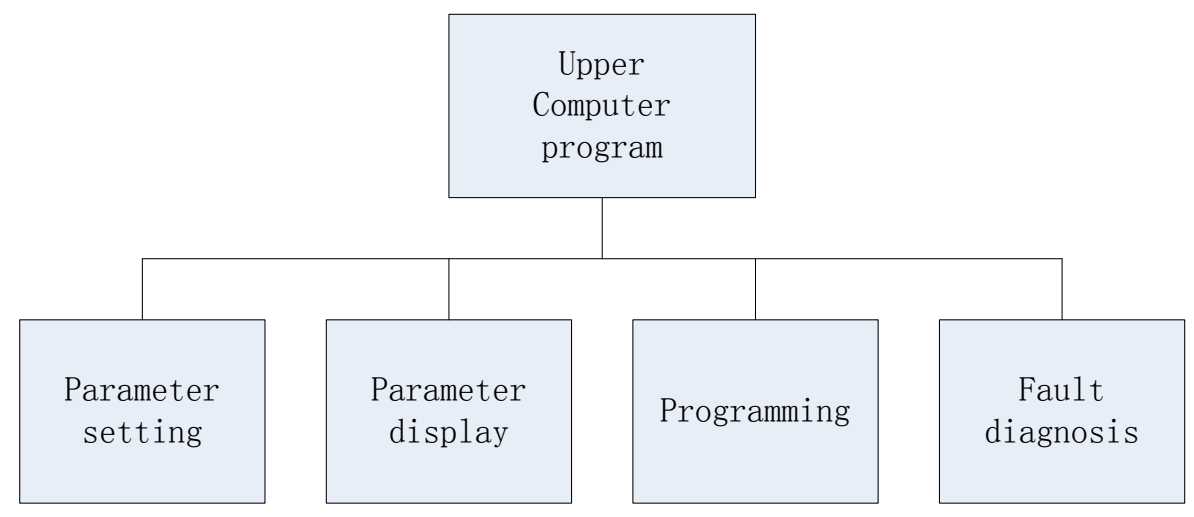

Figure5. The upper computer program

The lower computer program: The main function of the real-time program is on lower computer, as shown in Figure 5. The main function of the software is: parameter explaining, logic control, motion control and so on. Parameter explaining module is used to make the setting parameters convert into the program language that system can identify, and then processing; logic control is used to control the logic relationship between the variables, and to ensure system stability and orderly operation; motion control module is mainly according to the user's setting to control each roller of the plate rolling machine, rolling work is done through the coordinated movement between each roller.

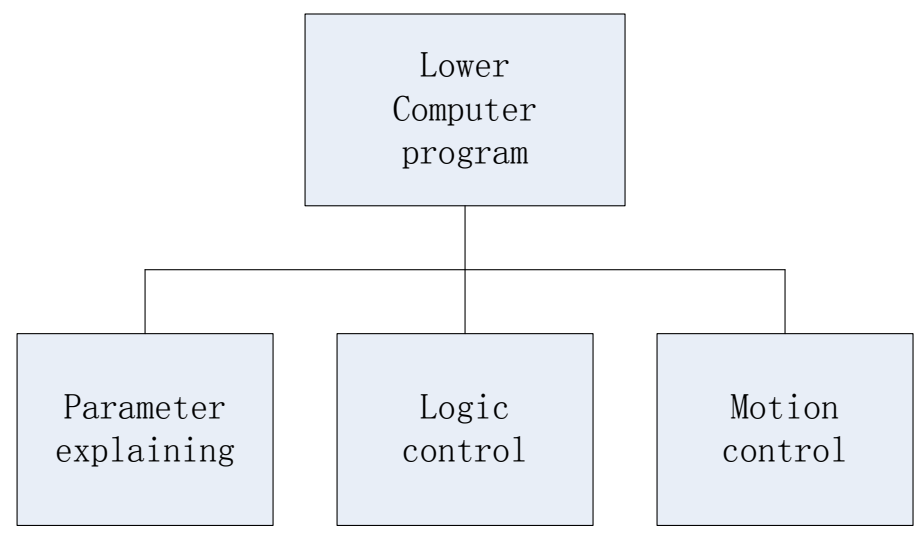

Figure6. The lower computer program

I/O design: The signal input and output of the system are accomplished by the I/O interface of the motion controller, including the feedback signal and the signal. The system I/O design is shown in Table 1.

Table1. System I/O design

\begin{tabular}{lccc}
\hline & Input & & Output \\
\hline IN0 & Upper roll horizontal signal & OUT0 & Indicator light 1 \\
IN1 & Upper roll vertical signal & OUT1 & Indicator light 2 \\
IN2 & Lower roll signal & OUT2 & Indicator light 3 \\
IN3 & Limiting signal 1 & OUT3 & Indicator light 4 \\
IN4 & Limiting signal 2 & OUT4 & Indicator light 5 \\
& & OUT5 & Indicator light 6 \\
\hline
\end{tabular}

\section{Summary}

In this paper, according to the requirement of the development of industrial automation, a kind of automatic rolling machine is designed. The system adopts the double CPU structure of the "PC+ motion controller”, which ensures the real-time and stability of the system, and improves the accuracy 
of the system, and the modular design accords with the idea of the open system. The hardware interface standardization and modularization design of hardware provides the expansion of space for the users, the users can according to the actual demand to increase the hardware, and the corresponding expansion of the software function. The system has a certain significance for the development of automatic plate rolling machine.

\section{Acknowledgement}

This research was financially supported by the National Science Foundation.

\section{References}

[1] Xing Weirong. The status and development of rolling machine [J]. Forging equipment and manufacturing technology, 2010, (2): 10-16.

[2] Liu Dehong. The domestic market status and forecast of hot rolled steel [J]. Steel, 2001, 18 (3): 41-42.

[3] Guo Yongping, Li Zewu. Commonly used three roller machine structure and transmission mode [J]. Forging and stamping equipment and manufacturing technology, 2010, 45 (6): 21-23.

[4] Gao Yaodong, where the snow. The dynamic simulation of the rolling process of bending machine [J]. China heavy equipment, 2010, (2): 4-7.

[5] Yang Shuping, Ma Yueshan. NC four roller bending machine achieving [J]. Journal of Shenyang Chemical University, 2005, 19 (2): 110-112. 